\title{
ALMOST NONPOSITIVELY CURVED MANIFOLDS
}

\author{
KENJI FUKAYA \& TAKAO YAMAGUCHI \\ Dedicated to Shingo Murakami on his sixtieth birthday
}

\section{Introduction}

Let $M$ be a compact connected $C^{\infty}$ Riemannian $n$-manifold with diameter $d(M) \leq D$. We say that $M$ has "almost nonpositive" curvature if the sectional curvature $K(M)$ satisfies $K(M)<\varepsilon$ for a small $\varepsilon>0$ depending on $D$ and $n$. In this paper, we study the topology of manifolds of almost nonpositive curvature under the condition $K(M) \geq-1$.

We denote by $\mathscr{M}(n, D)$ the family of compact Riemannian $n$-manifolds $M$ with $d(M) \leq D$ and $K(M) \geq-1$. The main result of this paper is the following.

Theorem 0.1. There exists a positive number $\varepsilon_{n}(D)$ such that the following holds. If $M \in \mathscr{M}(n, D)$ satisfies $K(M)<\varepsilon_{n}(D)$, then the universal covering space of $M$ is diffeomorphic to $\mathbf{R}^{n}$.

Theorem 0.1 was conjectured by Gromov [14, §4], where it is stated that the fundamental group $\pi_{1}(M)$ is infinite. One might hope to eliminate the condition $K(M) \geq-1$. But, for $n=3$, there is a counterexample due to Gromov [13, 1.6], which has been verified in a recent paper by Buser and Gromoll [3]: Namely, for given $\varepsilon>0$, there exists a metric $g_{\varepsilon}$ on the sphere $S^{3}$ such that $d\left(g_{\varepsilon}\right) \leq \varepsilon$ and $K\left(g_{\varepsilon}\right) \leq \varepsilon$.

In fact, we can prove a more precise result than Theorem 0.1 . To state it we need several notation. For $\alpha, 0<\alpha<1$, a $C^{1, \alpha}$ nonpositively curved orbifolds stands for a metric space $X / \Gamma$, where $X$ is a simply connected complete $C^{1, \alpha}$ Riemannian manifold of nonpositive curvature (in the sense of Definition 1.6), and $\Gamma$ is a properly discontinuous group of isometries of $X$. (Our terminology is a bit different from that in Thurston [22]. The orbifold in our sense is denoted as the good orbifold there.) We say that a map $f: M \rightarrow X / \Gamma$ is a fibration if $f$ has a lift $\tilde{f}: \tilde{M} \rightarrow X$ which is a fiber bundle, where $\tilde{M}$ is the universal covering space of $M$. The fiber of $f$ is the inverse image $f^{-1}(p)$ of a nonsingular point $p$ of 
$X / \Gamma$. In our case, this definition coincides with one in $[10, \S 7]$. We define the structure group of the fibration as in [10, $\S 7]$.

We consider a compact manifold $H / \Lambda$, where $H$ is a nilpotent Lie group, $\Lambda$ is a discrete subgroup of $H \tilde{\times} \operatorname{Aut}(H)$, and $[\Lambda: H \cap \Lambda]<\infty$. Put $\Lambda_{0}=\Lambda \cap H$. We take a series of subgroups $1=\Lambda^{(0)} \subset \Lambda^{(1)} \subset$ $\cdots \subset \Lambda^{(N)}=\Lambda_{0}$ such that $\Lambda^{(m)} / \Lambda^{(m-1)}$ is contained in the center of $\Lambda_{0} / \Lambda^{(m-1)}$. Let $L$ be a subgroup of $\operatorname{Aut}\left(\Lambda_{0}\right)$ containing $\operatorname{Int}\left(\Lambda_{0}\right)$. We assume that $\Lambda^{(m)}$ is preserved by the elements of $L$. Then we have a homomorphism $J: L \rightarrow \prod \operatorname{Aut}\left(\Lambda^{(m)} / \Lambda^{(m-1)}\right)=\prod \operatorname{SL}\left(k_{m} ; \mathbf{Z}\right)$. Remark that our assumption on $\Lambda^{(m)}$ implies that $J\left(\operatorname{Int}\left(\Lambda_{0}\right)\right)=1$.

Theorem 0.2. If $M \in \mathscr{M}(n, D)$ and $K_{M}<\varepsilon_{n}(D)$, then we have a fibration $H / \Lambda \rightarrow M \rightarrow X / \Gamma$ such that

(0.3.1) $X / \Gamma$ is a nonpositively curved orbifold of $C^{1, \alpha}$-class,

(0.3.2) $H$ is a nilpotent Lie group, $\Lambda \subset H \tilde{\times}$ Aut $H$, and $[\Lambda: \Lambda \cap H]<\infty$,

(0.3.3) the structure group of the fibration can be reduced to $C(H) /(C(H) \cap \Lambda) \tilde{x} L$, where $\operatorname{Int}\left(\Lambda_{0}\right) \subset L \subset \operatorname{Aut}\left(\Lambda_{0}\right)$, and

(0.3.4) we can choose $\Lambda^{(m)}$ so that $J(L)$ is a finite group.

Conversely we have the following.

Theorem 0.4. Let $H / \Lambda \rightarrow M \rightarrow X / \Gamma$ be a fibration satisfying (0.3.2)(0.3.4). Suppose that $X / \Gamma$ is a Riemannian orbifold of $C^{\infty}$-class. Then, for each $\varepsilon>0$, there exists a Riemannian metric $g_{\varepsilon}$ on $M$ such that:

$(0.5 .1) \varepsilon+\operatorname{Max}\left\{0, \sup K_{X}\right\}>K_{\left(M, g_{\varepsilon}\right)}>\operatorname{Min}\left\{0, \inf K_{X}\right\}-\varepsilon$,

(0.5.2) $\operatorname{diam}\left(M, g_{\varepsilon}\right)<\operatorname{diam}(X / \Gamma)+\varepsilon$,

(0.5.3) $\lim _{\varepsilon \rightarrow 0} d_{H}\left(\left(M, g_{\varepsilon}\right), X / \Gamma\right)=0$.

Remark 0.6. We do not know if $X / \Gamma$ in Theorem 0.2 admits a nonpositively curved metric of $C^{\infty}$-class.

Theorems 0.2 and 0.4 imply the following.

Corollary 0.7. If $M \in \mathscr{M}(n, D)$ and $K(M)<\varepsilon_{n}(D)$, then, for each $\varepsilon>0$, there exists a metric $g_{\varepsilon}$ on $M$ such that $\left(M, g_{\varepsilon}\right) \in \mathscr{M}(n, D)$ and $K\left(M, g_{\varepsilon}\right)<\varepsilon$.

Corollary 0.8. Let $M \in \mathscr{M}(n, D)$ and $K(M)<\varepsilon_{n}(D)$. Suppose that $\pi_{1}(M)$ is solvable. Then $M$ is diffeomorphic to $H / \Lambda$, where $H$ and $\Lambda$ are as in (0.3.2).

As an application of our argument to manifolds with almost nonnegative curvature, we have the following.

Theorem 0.9. Let $M \in \mathscr{M}(n, D)$ and $-\varepsilon_{n}(D)<K(M) \leq 1$. Suppose that $M$ is a $K(\pi, 1)$-space. Then $M$ is diffeomorphic to $H / \Lambda$, where $H$ and $\Lambda$ are as in (0.3.2). 
For the proof of our results, we make use of the theory of convergence and collapsing of Riemannian manifolds. Now we briefly sketch the idea of the proof of our theorems. We shall proceed by reduction to absurdity.

Let $\left(M_{i}, g_{i}\right), i=1,2, \cdots$, be a sequence in $\mathscr{M}(n, D)$ with $K\left(g_{i}\right)<$ $i^{-2}$ such that each $M_{i}$ does not satisfy the conclusion of Theorem 0.1 or 0.2 , and assume that $\left(M_{i}, g_{i}\right)$ converges to a metric space $Y$ for the Hausdorff distance. Since the maximal rank radius of the exponential mapping at a fixed point $m_{i}$ of $M_{i}$ is greater than $i$, the ball $B(i)$ of radius $i$ in $\mathbf{R}^{n}$ around the origin inherits an induced metric $\tilde{g}_{i}$. For a subsequence, $\left(B(i), \tilde{g}_{i}\right)$ converges to a $C^{1, \alpha}$ Riemannian manifold $X_{0}$ with respect to the pointed Hausdorff distance. The space $X_{0}$ has nonpositive curvature. Let $G_{i}$ be the fundamental pseudogroup of isometric imbeddings of $\left(B(i), \tilde{g}_{i}\right)$ into $\left(B(2 i), \tilde{g}_{i}\right)$ for the exponential mapping at $m_{i}$ such that $M_{i}$ is isometric to the quotient space $B(i) / G_{i}$. In a sense, $G_{i}$ converges to a group $G$ of isometries of $X_{0}$ such that $Y$ is isometric to $X_{0} / G$. The identity component $G_{0}$ of $G$ is a nilpotent Lie group, and contains no compact subgroups. Hence $G_{0}$ acts freely on $X_{0}$. It will turn out that $G_{0}$ acts by translations and $X_{0}$ splits isometrically as $X_{0}=X \times \mathbf{R}^{k}$, where the $\mathbf{R}^{k}$-factor is generated by $G_{0}$-orbits. Therefore, $Y$ is isometric to a $C^{1, \alpha}$ nonpositively curved orbifold $X / \Gamma$, where $\Gamma=G / G_{0}$. By passing to the orthonormal frame bundle of $M_{i}$, we shall overcome the difficulty in dealing with singular points of $X / \Gamma$, and construct a fibration $M_{i} \rightarrow X / \Gamma$ for large $i$. We can use this fibration to prove Theorem 0.1 . More detailed study of our fibration implies Theorem 0.2.

The organization of this paper is as follows. The constructions of $X_{0}$ and $G$ are done in $\S 1$ and $\S 2$ respectively. $\S 3$ is devoted to the proof of the properties of $G_{0}$ and the splitting of $X_{0}$. The proof of Theorem 0.1 is deferred to $\S 4$, where the deduction of Corollary 0.8 from Theorems 0.2 and 0.4 also appears. In $\S 5$, we shall rewrite the condition (0.3.4) in Theorem 0.2 in terms of the homotopy exact sequence of the fibration $M \rightarrow X / \Gamma$. After preliminary arguments in $\S 6$, we shall construct in $\S 7$ the subgroups $\Lambda^{(i)} \subset \Lambda$ in (0.3.4). The proof of Theorem 0.2 is completed in $\S 8$. $\S 9$ is devoted to the proof of Theorem 0.4 . In $\S 10$, we shall prove Theorem 0.9 .

\section{Basic properties of the space $X_{0}$}

For a positive number $r$ and a point $x$ in a metric space $X$, we denote by $B_{r}(x, X)$ the metric $r$-ball around $x$. For $X=\mathbf{R}^{n}$, we set $B(r)$ instead of $B_{r}\left(0, \mathbf{R}^{n}\right)$ for simplicity. 
Let $M_{i}, i=1,2, \cdots$, be a sequence in the family $\mathscr{M}(n, D)$ such that $K\left(M_{i}\right)<i^{-2}$. Let $f_{i}: \mathbf{R}^{n} \rightarrow M_{i}$ be the composition of a linear isometry of $\mathbf{R}^{n}$ onto the tangent space $T_{m_{i}}\left(M_{i}\right)$ and the exponential map at a fixed point $m_{i}$ of $M_{i}$. By the Rauch comparison theorem, $f_{i}$ has maximal rank on the ball $B(2 i)$. Let $\tilde{g}_{i}$ be the induced metric $f_{i}^{*} g_{i}$ on $B(2 i)$, where $g_{i}$ is the metric of $M_{i}$. Remark that $B(i)$ is a convex set of $\left(B(2 i), \tilde{g}_{i}\right)$. By $[15,8.23]$, passing to a subsequence if necessary, the pointed space $\left(B(2 i), \tilde{g}_{i}, 0\right)$ converges to a pointed $C^{1, \alpha}$ Riemannian $n$-manifold $\left(X_{0}, x_{0}\right)$ with respect to the pointed Hausdorff distance.

In this section, we study basic geometric properties of $X_{0}$ needed in subsequent sections. To do this, we use the center of mass technique, and obtain an imbedding $\varphi_{i}: B(i) \rightarrow B(2 i)$ such that the induced metric $\hat{g}_{i}=\varphi_{i}^{*} \tilde{g}_{i}$ converges to a $C^{1, \alpha}$ metric $\hat{g}_{\infty}$ of $X_{0}$ on $B(R)$ for each $R>0$ [11], [20].

First of all, we note that there is a unique $\hat{g}_{\infty}$-geodesic which satisfies a given initial condition, and that at each point, the $\hat{g}_{i}$-exponential map converges to that of $X_{0}$ uniformly on each compact subset in the tangent space. By $d$ and $d_{i}$ we denote the distance defined by using the metrics $\hat{g}_{\infty}$ and $\hat{g}_{i}$ respectively.

The space $X_{0}$ has "nonpositive curvature" in the following sense.

Lemma 1.1. Let $\sigma, \tau:[0,1] \rightarrow X_{0}$ be geodesic segments in $X_{0}$ with length $a, b$ respectively such that $\sigma(0)=\tau(0)$, and let $\alpha$ be the angle between them. Then, we have

$$
d(\sigma(1), \tau(1)) \geq a^{2}+b^{2}-2 a b \cos \alpha .
$$

Proof. Let $\sigma_{i}$ and $\tau_{i}$ be $\hat{g}_{i}$-geodesics such that $\sigma_{i}^{\prime}(0)=\sigma^{\prime}(0)$ and $\dot{\tau}_{i}(0)=\dot{\tau}(0)$ for large $i$. Let $\bar{\sigma}_{i}$ and $\bar{\tau}_{i}$ be geodesics on the sphere of constant curvature $i^{-2}$ such that $\bar{\sigma}_{i}(0)=\bar{\tau}_{i}(0),\left|\sigma_{i}^{\prime}(0)\right|=\left|\bar{\sigma}_{i}^{\prime}(0)\right|$, $\left|\tau_{i}^{\prime}(0)\right|=\left|\bar{\tau}_{i}^{\prime}(0)\right|$, and that the angle between them is equal to $\alpha$. Then the Rauch comparison theorem implies $d_{i}\left(\sigma_{i}(1), \tau_{i}(1)\right) \geq d\left(\bar{\sigma}_{i}(1), \bar{\tau}_{i}(1)\right)$. Taking the limit, we have the required inequality.

As immediate consequences of Lemma 1.1, we have the following lemmas.

Lemma 1.2. For any two points in $X_{0}$, there is a unique geodesic of $X_{0}$ joining them.

Lemma 1.3. The distance function $d: X_{0} \times X_{0} \rightarrow \mathbf{R}$ is convex.

Proof. We have only to show that for geodesics $\sigma$ and $\tau$ of $X_{0}$ defined on a bounded interval $I$, the function $t \rightarrow d(\sigma(t), \tau(t))$ is convex on $I$. 
Let $\sigma_{i}$ and $\tau_{i}$ be $\hat{g}_{i}$-geodesics with $\sigma_{i} \rightarrow \sigma$ and $\tau_{i} \rightarrow \tau$. We set

$$
f(t)=d(\sigma(t), \tau(t)), \quad f_{i}(t)=d_{i}\left(\sigma_{i}(t), \tau_{i}(t)\right) .
$$

For every fixed $t_{0}$ in $I$, set $l_{i}=d_{i}\left(\sigma_{i}\left(t_{0}\right), \tau_{i}\left(t_{0}\right)\right)$. Let $\alpha_{i}(s, t)$ be a variation such that for each $t$, the curve $s \rightarrow \alpha_{i}(s, t) \quad\left(0 \leq s \leq l_{i}\right)$ is a $\hat{g}_{i}$-geodesic from $\sigma_{i}(t)$ to $\tau_{i}(t)$. By the second variation formula, we have

$$
\begin{aligned}
f_{i}^{\prime \prime}\left(t_{0}\right) & =\int_{0}^{l_{i}} \hat{g}_{i}\left(V_{i}^{\prime}, V_{i}^{\prime}\right)-K\left(V_{i} \wedge T_{i}\right)\left\|V_{i} \wedge T_{i}\right\|^{2}-\hat{g}_{i}\left(V_{i}^{\prime}, T_{i}\right) d s \\
& =\int_{0}^{l_{i}}\left\|V_{i}^{\prime} \wedge T_{i}\right\|^{2}-K\left(V_{i} \wedge T_{i}\right)\left\|V_{i} \wedge T_{i}\right\|^{2} d s,
\end{aligned}
$$

where $V_{i}=\partial \alpha_{i} / \partial t, T_{i}=\partial \alpha_{i} / \partial s$, and $K\left(V_{i} \wedge T_{i}\right)$ denotes the sectional curvature of the plane section $V_{i} \wedge T_{i}$ with respect to $\hat{g}_{i}$. The curvature assumption and a standard estimate on Jacobi fields imply $f_{i}^{\prime \prime}\left(t_{1}\right) \geq-C / i^{2}$ for some constant $C$. This yields the inequality

$$
f_{i}(t) \geq f_{i}\left(t_{0}\right)+f_{i}^{\prime}\left(t_{0}\right)\left(t-t_{0}\right)-C\left(t-t_{0}\right)^{2} / 2 i^{2}
$$

for all $t$ in $I$. Taking the limit, we obtain that $f(t) \geq f\left(t_{0}\right)+\beta\left(t-t_{0}\right)$, where $\beta$ is the limit of $f_{i}^{\prime}\left(t_{0}\right)$ which exists certainly by the first variation formula. This shows the convexity of $f$.

Lemma 1.4. Let $A$ be a closed convex set of $X_{0}$. Then we have the following:

(1.5.1) For each $x$ in $X_{0}$, there exists a unique point $\rho(x)$ in $A$ satisfying $d(x, \rho(x))=d(x, A)$.

(1.5.2) The mapping $\rho: X_{0} \rightarrow A$ is distance-nonincreasing.

Proof. (1.5.1) Suppose that for a point $x$ there exist distinct points $y_{1}$ and $y_{2}$ of $A$ such that $d\left(x, y_{i}\right)=d(x, A), i=1,2$. Let $\sigma_{i}$ and $\tau$ be geodesics joining $x$ to $y_{i}$ and $y_{1}$ to $y_{2}$ respectively. We note that, by Lemma 1.1, the sum of interior angles of a geodesic triangle in $X_{0}$ is less than or equal to $\pi$. Since $A$ is convex, $\sigma_{i}$ and $\tau$ make obtuse angles. This is a contradiction.

(1.5.2) For $x_{1}$ and $x_{2}$ in $X_{0}$, let $\sigma_{i}:[0,1] \rightarrow X_{0}, i=1,2$, be the geodesics from $\rho(x)$ to $x_{i}$. Since $\sigma_{i}$ and the geodesic joining $\rho\left(x_{1}\right)$ and $\rho\left(x_{2}\right)$ make obtuse angles, the argument in the proof of Lemma 1.3 applied to the function $f(t)=d\left(\sigma_{1}(t), \sigma_{2}(t)\right)$ yields $d\left(x_{1}, x_{2}\right) \geq d\left(\rho\left(x_{1}\right), \rho\left(x_{2}\right)\right)$.

Definition 1.6. We say that a complete Riemannian manifold of $C^{1, \alpha}$ class has nonpositive curvature, if its universal covering space satisfies the conclusions of Lemmas 1.1-1.4. 


\section{The construction of the group $G$}

We consider the sequence of Riemannian manifolds $\left(B(i), \hat{g}_{i}\right)$, constructed in $\S 1$. Put $D_{i}=d_{i}(0, \partial B(i))$, remark that $\lim _{i \rightarrow \infty} D_{i}=\infty$, and set $B_{i}(R)=\left\{p \in B(i) \mid d_{i}(0, p)<R\right\}$. When $D_{i}>2 R$, we define

$$
G_{i}^{\prime}(R)=\left\{\psi: B_{i}(R) \rightarrow B_{i}(2 R) \mid \psi \text { is continuous and } f_{i} \varphi_{i} \psi=f_{i} \varphi_{i}\right\},
$$

where $f_{i}: \mathbf{R}^{n} \rightarrow M_{i}$ and $\varphi_{i}: B(i) \rightarrow B(2 i)$ are the maps defined in $\S 1$. Remark that $\psi^{*} \hat{g}_{i}=\hat{g}_{i}$ for $\psi \in G_{i}^{\prime}(R)$. Since $B_{i}(2 R)$ converges to $B_{2 R}\left(x_{0}, X_{0}\right)$, we can regard $B_{i}(2 R)$ as a subset of $X_{0}$. For each $R$, there exists $i_{0}$ such that $B_{i}(2 R) \supset B_{R}\left(x_{0}, X\right)$ for $i>i_{0}$. By $G_{i}(R)$, we denote the set of the restrictions of the elements of $G_{i}^{\prime}(2 R)$ to $B_{R}\left(x_{0}, R\right)$. We have

$$
\frac{1}{2}<\frac{d(\psi(p), \psi(q))}{d(p, q)}<2
$$

for $p, q \in B_{R}\left(x_{0}, X_{0}\right)$, and $i>i(R)$, since $\left(B_{R}\left(x_{0}, X\right), d_{i}\right)$ converges to $\left(B_{R}\left(x_{0}, X\right), d\right)$ with respect to the Lipschitz distance, and the elements of $G_{i}(R)$ are isometries for $d_{i}$. In other words $G_{i}(R)$ is contained in the set

$$
L(R)=\left\{\psi: B_{R}\left(x_{0}, X\right) \rightarrow B_{4 R}\left(x_{0}, X\right) \mid \frac{1}{2} \leq \frac{d(\psi(p), \psi(q))}{d(p, q)} \leq 2\right\} .
$$

We define a metric on $L(R)$ by

$$
d\left(\psi, \psi^{\prime}\right)=\sup \left\{d\left(\psi(p), \psi^{\prime}(p)\right) \mid p \in B_{R}\left(x_{0}, X\right)\right\} .
$$

Ascoli-Arzela's Theorem implies the compactness of $(L(R), d)$. Therefore, we may assume, by taking a subsequence if necessary, that $G_{i}(R)$ converges to a subset $G(R)$ with respect to the Hausdorff distance in $L(R)$. Set $R<R^{\prime}$. Since $f_{i} \varphi_{i}$ is of maximal rank on the ball of radius $D_{i}$, it follows that every element of $G_{i}(R)$ is a restriction of an element of $G_{i}\left(R^{\prime}\right)$ if $i$ is sufficiently large. Hence we have an injective homomorphism $I_{R}^{R^{\prime}}: G_{i}(R) \rightarrow G_{i}\left(R^{\prime}\right)$ such that $I_{R}^{R^{\prime}}(\psi)=\psi$ on $B_{R}\left(x_{0}, X\right)$. These maps induce an inclusion $I: G(R) \rightarrow G\left(R^{\prime}\right)$. We put $\bigcup_{R} G(R)=G$. It is easy to see that $G$ is a group of isometries of $(X, d)$. We put a compact open topology on $G$.

Lemma 2.1. $X / G$ is isometric to $Y$, the limit of $M_{i}$.

The proof is a pseudogroup version of [6, Theorem 2-1] and is left to the reader. 
Lemma 2.2. The connected component $G_{0}$ of $G$ is a Lie group. The quotient group $G / G_{0}$ equipped with the quotient topology is discrete.

Proof. Remark that $G$ is a closed subgroup of the group of all isometries of $X$. Hence, in the case when the metric $\hat{g}_{\infty}$ on $X$ is of $C^{\infty}$-class, the conclusion follows immediately from [18, Theorem 3.4]. In the general case, we can use [2] in a way similar to [8, §1] to obtain a smooth Riemannian $G$-manifold $\left(X_{0}^{\prime}, \hat{g}_{\infty}^{\prime}\right)$ such that $\left(X_{0}, G\right)$ and $\left(X_{0}^{\prime}, \hat{g}_{\infty}^{\prime}\right)$ are equivariantly diffeomorphic. Therefore the lemma follows immediately from the case where $\hat{g}_{\infty}$ is smooth.

Lemma 2.3. $\quad G_{0}$ is nilpotent.

This is a consequence of Margulis' lemma. The proof is similar to [8, $\S 4]$, and hence is omitted.

\section{Splitting $X_{0}$ to a direct product}

In this section, we shall prove that $X_{0} / G$ is a Riemannian orbifold of nonpositive curvature. We shall work under the following conditions.

(3.1.1) $X_{0}$ is a complete and simply connected $C^{1, \alpha}$ Riemannian manifold satisfying Lemmas 1.1-1.4.

(3.1.2) $G$ is a group of isometries of $X_{0}$, and $X_{0} / G$ is compact.

(3.1.3) $G / G_{0}$ is discrete, where $G_{0}$ is the connected component of $G$.

(3.1.4) $G_{0}$ is a nilpotent Lie group.

For $g \in G$ and $p \in X$ we put $\delta_{g}(p)=d(p, g(p))$. For $A \subset G$, we define

$$
C_{A} X_{0}=\left\{p \in X \mid \delta_{g}(p)=\inf _{x \in X} \delta_{g}(x) \text { for every } g \in A\right\}
$$

We take a sequence of subgroups $G^{(i)}$ of $G_{0}$ such that

(3.1.5.1) $G^{(0)}$ is trivial and $G^{(N)}=G_{0}$,

(3.1.5.2) $G^{(i)}$ is normal in $G$, and $G^{(i+1)} / G^{(i)}$ is the center of $G_{0} / G^{(i)}$.

Lemma 3.2. Assume (3.1.1)-(3.1.5). Then :

(3.2.1) $C_{G_{0}} X_{0}=X_{0}$,

(3.2.2) $G_{0}$ is isomorphic to $\mathbf{R}^{n-m}$,

(3.2.3) $X_{0}$ is isometric to a direct product $X \times \mathbf{R}^{n-m}$, where $X$ is a $C^{1, \alpha}$ Riemannian manifold,

(3.2.4) for $(x, a) \in X \times \mathbf{R}^{n-m}=X_{0}$ and $b \in \mathbf{R}^{n-m}$, we have $b(x, a)=$ $(x, a+b)$.

Proof. The proof is by induction on $\operatorname{dim} X_{0}$.

Sublemma 3.3. For each $g \in G^{(1)}$, the set $C_{\{g\}} X_{0}$ is nonempty. 
Proof. Put $A=\left\{h^{-1} g h \mid h \in G\right\}$. Since $G^{(1)}$ is the center of $G_{0}$, and $G / G_{0}$ is discrete, it follows that $A$ is discrete. Put

$$
\delta(p)=\inf \{d(p, f(p)) \mid f \in A\} .
$$

In view of the compactness of $X_{0} / G$ and the $G$-invariance of $A$, the function $\delta$ assumes its minimum at some point $p$. Then the discreteness of $A$ implies that there exists $h \in G$ such that $p \in C_{\left\{h^{-1} g h\right\}} X_{0}$. Therefore $h(p)$ is contained in $C_{\{g\}} X_{0}$.

Let $G^{\prime} \subset G_{0}$ denote the set of all semisimple elements of $G_{0}$. (Here we recall that the isometry $\gamma$ is said to be semisimple if $C_{\{\gamma\}} X_{0}$ is nonempty.) Sublemma 3.3 implies that $G^{\prime} \neq\{1\}$. On the other hand, $[1$, p. 88, Lemma] implies that

(3.4.1) $G^{\prime}$ is a normal subgroup of $G_{0}$,

(3.4.2) $C_{G^{\prime}} X_{0}$ is nonempty,

(3.4.3) $C_{G^{\prime}} X_{0}$ splits as $X^{\prime} \times \mathbf{R}^{s}$,

(3.4.4) $G^{\prime}$ is isomorphic to $\mathbf{R}^{s}$ and it acts on $C_{G^{\prime}} X_{0}$ as the translation of the second factor.

Remark that the proof in [1] uses only the properties which we proved in $\S 1$, and hence it can be applied to our case, where the metric is not smooth.

It is easy to see that $G^{\prime}$ is also a normal subgroup of $G$, so that $C_{G^{\prime}} X_{0}$ is a $G$-invariant subset. Since $C_{G^{\prime}} X_{0}$ is convex and $X_{0} / G$ is compact, it follows that $X_{0}=C_{G^{\prime}} X_{0}$. Hence $X_{0}=X^{\prime} \times \mathbf{R}^{s}$. Since $G^{\prime}$ is a normal subgroup of $G$ and the $\mathbf{R}^{s}$ factor is generated by $G^{\prime}$-action, the splitting of $X_{0}$ is preserved by $G$-action. Therefore $G / G^{\prime}$ acts on $X^{\prime}$ by isometry, and this action satisfies (3.1.1)-(3.1.5). We apply the induction hypothesis to this action and conclude:

(3.5.1) $G_{0} / G^{\prime} \simeq \mathbf{R}^{t}$,

(3.5.2) $X^{\prime}=X \times \mathbf{R}^{t}$,

(3.5.3) $G_{0} / G^{\prime}$ acts on $X^{\prime}$ as translation of the second factor.

Therefore we have:

(3.6.1) $X_{0}=X \times \mathbf{R}^{t+s}$,

(3.6.2) $G$ preserves the splitting,

(3.6.3) the action of $G_{0}$ on the first factor, $X$, is trivial,

(3.6.4) the action of $G_{0}$ on the second factor is free.

(3.2.1)-(3.2.4) follow immediately.

Lemma 3.7. $X$ is diffeomorphic to the Euclidean space.

Proof. Fix a point $p_{0}$ on $X$. Take a neighborhood $U$ of $p_{0}$, which is diffeomorphic to the Euclidean space. Take a smooth function on $X$, 
which is equal to 1 outside $U$ and vanishes in a neighborhood of $p_{0}$. For each $p \in X$, let $V(p) \in T_{p}(X)$ be the unit vector tangent to the unique minimal geodesic connecting $p$ and $p_{0} . f V$ is a vector field of $C^{0}$-class. We take a smooth approximation of $f V$, and let $\Phi_{t}$ be the oneparameter group of transformations associated to the vector field. Then, for each compact subset $K$ of $X$, there exists $t$ such that $\Phi_{-t}(U) \supset K$. The lemma follows immediately.

Finally we remark that the following lemma is proved in a way similar to the $C^{\infty}$-case proved in [12], [19].

Lemma 3.8. Let $X$ be a complete and simply connected $C^{1, \alpha}$ manifold with nonpositive curvature, and let $\Gamma$ be a properly discontinuous group of isometries of $X$. Assume that $\Gamma$ is solvable and that, for each $\gamma \in \Gamma$, the set $C_{\{\gamma\}} X$ is nonempty. Then we have a subgroup $\Gamma^{\prime}$ of $\Gamma$ such that

(3.8.1) $\left[\Gamma: \Gamma^{\prime}\right]<\infty$,

(3.8.2) $\Gamma^{\prime}$ is a free abelian,

(3.8.3) there exists a totally geodesic flat $\Gamma^{\prime}$-invariant subspace $Z$ of $X$ such that $Z / \Gamma^{\prime}$ is compact.

\section{Proof of Theorem 0.1}

In $\S \S 1-3$, we proved that $\left(M_{i}, g_{i}\right)$ converges to $\left(\mathbf{R}^{m}, \bar{g}\right) / \Gamma$ for the Hausdorff distance, where $\bar{g}$ is a $C^{1, \alpha}$ metric and $\Gamma$ is a properly discontinuous group of isometries. We recall the results of [8] here. Let $F M_{i}$ be the set of all orthonormal frames of $M_{i}$. In an obvious way, $g_{i}$ induces a metric $h_{i}$ on $F M_{i}$. By taking a subsequence if necessary, we may assume that $\left(F M_{i}, h_{i}\right)$ converges with respect to the Hausdorff distance. It is easy to see that the limit is isometric to $\left(F X, h_{\infty}\right) / G$, where $h_{\infty}$ is a metric induced from the metric $\hat{g}_{\infty}$ on $X$. Since $F X \simeq \mathbf{R}^{n} \times \mathrm{O}(n), G / G_{0} \simeq \Gamma$, and $G_{0} \simeq \mathbf{R}^{n-m}$, it follows that $F X / G$ is isometric to $\left(\mathbf{R}^{m} \times \mathrm{O}(n)\right) / \Gamma$. By $[8, \S 6]$, we see that the action of $\Gamma$ on $\mathbf{R}^{m} \times \mathrm{O}(n)$ is free. By $[8, \S 10]$, there exists the following commutative diagram:

$$
\begin{aligned}
& F M_{i} \underset{\tilde{\pi}_{i}}{\longrightarrow}\left(\mathbf{R}^{m} \times \mathrm{O}(n)\right) / \Gamma \\
& \downarrow / \mathrm{O}(n) \quad \downarrow / \mathrm{O}(n) \\
& M_{i} \underset{\pi_{i}}{\longrightarrow} \quad \mathbf{R}^{m} / \Gamma
\end{aligned}
$$

Here the map $\tilde{\pi}_{i}$ is a fibration (in the usual sense). Moreover, $\tilde{\pi}_{i}$ is an 
$\mathrm{O}(n)$-map. Now we put

$$
\begin{gathered}
F E_{i}=\left\{(q, y) \in F M_{i} \times\left(\mathbf{R}^{m} \times \mathrm{O}(n)\right) \mid \tilde{\pi}_{i}(q) \equiv y \bmod \Gamma\right\}, \\
E_{i}=F E_{i} / \mathrm{O}(n) .
\end{gathered}
$$

Remark that $\mathrm{O}(n)$ acts freely on $F E_{i}$. Then there exists the following commutative diagram:

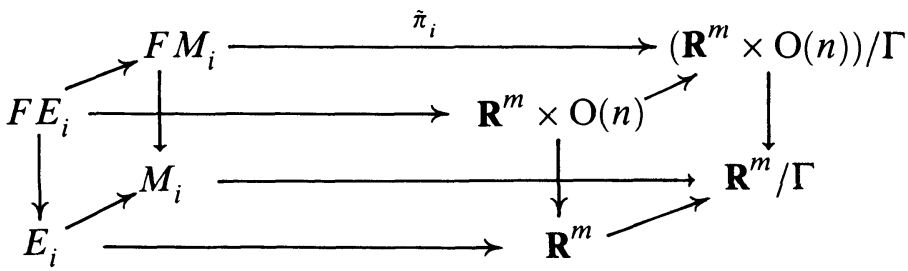

Since $\tilde{\pi}_{i}$ is a fibration, so is the map $F E_{i} \rightarrow \mathbf{R}^{m} \times \mathrm{O}(n)$. Hence $F E_{i} \rightarrow \mathbf{R}^{m}$ is a submersion, and $E_{i} \rightarrow \mathbf{R}^{m}$ is a fibration. Since $F E_{i} \rightarrow \mathbf{R}^{m} \times \mathrm{O}(n)$ is also an $\mathrm{O}(n)$-map, the fiber of $E_{i} \rightarrow \mathbf{R}^{m}$ is equal to that of $F M_{i} \rightarrow$ $\left(\mathbf{R}^{m} \times \mathrm{O}(n)\right) / \Gamma$, and is therefore diffeomorphic to an infranilmanifold. Thus that $E_{i}$ is diffeomorphic to the product of the Euclidean space and an infranilmanifold.

Since $\mathbf{R}^{m} \times \mathrm{O}(n) \rightarrow\left(\mathbf{R}^{m} \times \mathrm{O}(n)\right) / \Gamma$ is a covering map, so is the map $F E_{i} \rightarrow F M_{i}$. Hence $F E_{i} \rightarrow M_{i}$ is a submersion, and $E_{i} \rightarrow M_{i}$ is a covering map. Thus, we conclude that the universal covering space of $M_{i}$ is diffeomorphic to one of $E_{i}$, which is the Euclidean space. The proof of Theorem 0.1 is complete.

\section{Preliminary discussion on fundamental groups and structure groups}

To prove Theorem 0.2 , we rewrite condition $(0.3 .4)$ in terms of the homotopy exact sequence of the fibration. For this purpose, we recall several facts concerning reductions of the structure groups of fibrations. Let $F$ be a topological space and let $H(F)$ be the group of homeomorphisms equipped with compact open topology. We shall define a homomorphism $\Phi: H(F) / H_{0}(F) \rightarrow \operatorname{Aut}\left(\pi_{1}(F)\right) / \operatorname{Int}\left(\pi_{1}(F)\right)$, where $H_{0}(F)$ stands for the connected component of $H(F)$, and $\operatorname{Aut}\left(\pi_{1}(F)\right)$ and $\operatorname{Int}\left(\pi_{1}(F)\right)$ are the groups of all automorphisms and all inner automorphisms of the fundamental group of $F$, respectively. Fix $p_{0} \in F$ and $\varphi \in H(F)$. Then we have $\varphi_{*}: \pi_{1}\left(F, p_{0}\right) \rightarrow \pi_{1}\left(F, \varphi\left(p_{0}\right)\right)$. Using a path connecting $p_{0}$ and $\varphi\left(p_{0}\right)$, we have an isomorphism $\pi_{1}\left(F, \varphi\left(p_{0}\right)\right) \rightarrow \pi_{1}\left(F, p_{0}\right)$. If we change the choice of the path, the isomorphism changes by an element of $\operatorname{Int}\left(\pi_{1}\left(F, p_{0}\right)\right)$. Thus we have a homomorphism $\tilde{\Phi}: H(F) \rightarrow$ $\operatorname{Aut}\left(\pi_{1}(F)\right) / \operatorname{Int}\left(\pi_{1}(F)\right)$. Clearly $\tilde{\Phi}$ induces a map $\Phi$. 
Next, let $F \rightarrow M \stackrel{\pi}{\rightarrow} Y$ be a smooth fiber bundle with structure group $G$, a Lie group. Then, using the holonomy of a $G$-connection, we obtain a homomorphism $\Psi: \pi_{1}(Y) \rightarrow G / G_{0}$, where $G_{0}$ is the connected component of $G$. Let $I: G / G_{0} \rightarrow H(F) / H_{0}(F)$ be the homomorphism induced by the action of $G$ on $F$. Thus, we obtain a homomorphism $\Phi I \Psi: \pi_{1}(Y) \rightarrow \operatorname{Aut}\left(\pi_{1}(F)\right) / \operatorname{Int}\left(\pi_{1}(F)\right)$. We shall describe this homomorphism in terms of the homotopy exact sequence of the fibration. For simplicity, we assume $\pi_{0}(F)=\pi_{2}(Y)=\{1\}$. Then we have a short exact sequence

$$
1 \rightarrow \pi_{1}(F) \rightarrow \pi_{1}(M) \stackrel{\pi_{*}}{\longrightarrow} \pi_{1}(Y) \rightarrow 1 .
$$

Let $\gamma \in \pi_{1}(Y)$. Choose $\tilde{\gamma} \in \pi_{1}(M)$ satisfying $\pi_{*}(\tilde{\gamma})=\gamma$. We can define an element $\operatorname{conj}(\tilde{\gamma}) \in \operatorname{Aut}\left(\pi_{1}(F)\right)$ by $\operatorname{conj}(\tilde{\gamma})(\mu)=\tilde{\gamma}^{-1} \mu \tilde{\gamma}$. The equivalence class in $\operatorname{Aut}\left(\pi_{1}(F)\right) / \operatorname{Int}\left(\pi_{1}(F)\right)$ of the element conj $(\tilde{\gamma})$ is independent of the choice of $\tilde{\gamma}$ and depends only on $\gamma$. Thus, we obtain a homomorphism conj: $\pi_{1}(Y) \rightarrow \operatorname{Aut}\left(\pi_{1}(F)\right) / \operatorname{Int}\left(\pi_{1}(F)\right)$, and also have

Lemma 5.1. $\operatorname{conj}=\Phi I \Psi$.

We omit the proof.

Thirdly, we recall the following result (see [18, pp. 83-91]).

Lemma 5.2. Let $G^{\prime}$ be a subgroup of $G$ such that $\Psi\left(\pi_{1}(Y)\right)=G^{\prime} / G_{0}$. Then the structure group of the fibration $F \rightarrow M \rightarrow Y$ can be reduced to $G^{\prime}$.

Fourthly we remark that the preceding arguments can be applied also to the singular fibration $F \rightarrow M \rightarrow X / \Gamma$ which we study in the preceding sections. (We replace $\pi_{1}(Y)$ by $\Gamma$.) For example, the homotopy exact sequence is obtained by applying the nine-lemma to the following commutative diagram:

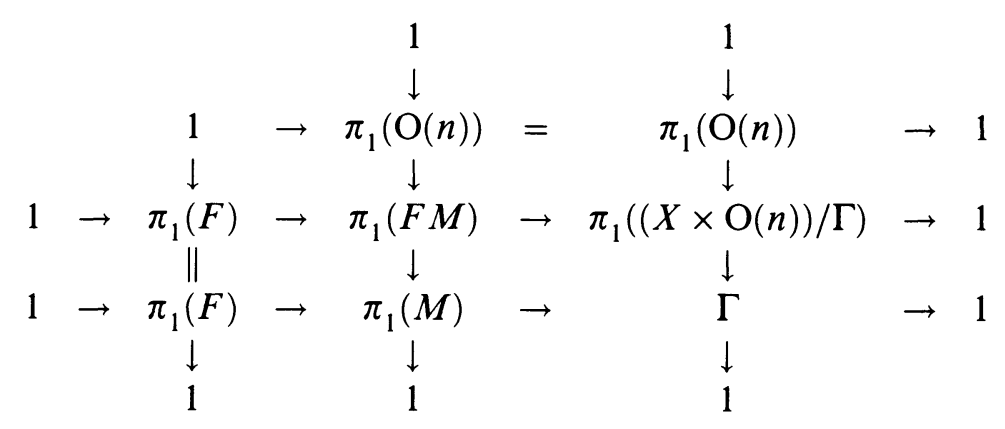

Now, we can rewrite $(0.3 .4)$ in terms of the fundamental groups. (Remark that the result of $\S 4$ and [10] imply (0.3.1)-(3.3.3).) Let $H / \Lambda \rightarrow$ $M \rightarrow X / \Gamma$ satisfy conditions $(0.3 .1),(0.3 .2)$, and (0.3.3). Then, $\Lambda$ is 
a normal subgroup of $\pi_{1}(M)$. We define a homomorphism $\pi_{1}(M) \rightarrow$ $\operatorname{Aut}(\Lambda)$ by $\gamma \rightarrow\left(\mu \rightarrow \gamma^{-1} \mu \gamma\right)$. In the case when subgroups $\Lambda^{(m)}, m=$ $1,2, \cdots$, are normal in $\pi_{1}(M)$, we can compose this map and $J: L \rightarrow$ $\prod \operatorname{SL}\left(k_{m} ; \mathbf{Z}\right)$, to obtain a homomorphism $\pi_{1}(M) \rightarrow \prod \operatorname{SL}\left(k_{i} ; \mathbf{Z}\right)$. We denote this map also by $J$. Then, Lemmas 5.1 and 5.2 imply the following.

Lemma 5.3. (0.3.4) is equivalent to the following:

(5.4) We can choose $\Lambda^{(m)}$ so that $J\left(\pi_{1}(M)\right)$ finite.

\section{Limit of universal covering spaces}

Let $M_{i}, i=1,2, \cdots$, be a sequence in $\mathscr{M}(n, D)$ with $K\left(M_{i}\right)<i^{-2}$. We study the limit space of the universal covering space $\tilde{M}_{i}$ of $M_{i}$. Fix a point $x_{i}$ and $\tilde{M}_{i}$. By taking a subsequence if necessary, we may assume that $\left(\tilde{M}_{i}, x_{i}\right)$ converges to a pointed metric space $(\tilde{X}, \tilde{x})$ for the pointed Hausdorff distance. In this section, we shall prove the following lemma which is needed in the next section.

Lemma 6.1. $\quad \tilde{X}$ is isometric to the space $X_{0}$ constructed in $\S 1$.

Since $M_{i}$ is a $K(\pi, 1)$-space by our Theorem 0.1 , Lemma 3.2 in [9] shows that $\tilde{X}$ has dimension $n$. By $[15,8.39]$, the injectivity radius at $x_{i}$ is uniformly bounded away from zero. Thus Lemma 4.2 of [9] implies that $\tilde{X}$ is contractible.

For the proof of Lemma 6.1, we need the following.

Lemma 6.2. For each point $x$ in $\tilde{X}$, the exponential map $\exp _{X}^{\infty}$ of $\tilde{X}$ is bijective.

When the metric of $\tilde{X}$ is of class $C^{2}$, Lemma 6.2 is nothing but the Cartan-Hadamard Theorem. But, since the metric of $\tilde{X}$ is only of class $C^{1, \alpha}$, it is a priori nontrivial that the exponential map is even a local homeomorphism.

For the point $x \in \tilde{X}$, take $y_{i} \in \tilde{M}_{i}$ so that $\left(\tilde{M}_{i}, y_{i}\right)$ converges to $(\tilde{X}, x)$ for the pointed Hausdorff distance. To prove Lemma 6.2, we use a center of mass technique to obtain an imbedding $\varphi_{i}: B_{3 i}(x, \tilde{X}) \rightarrow$ $B_{4 i}\left(y_{i}, \tilde{M}_{i}\right)$ such that the induced metric $\hat{g}_{i}=\varphi_{i}^{*} \tilde{g}_{i}$, where $\tilde{g}_{i}$ is the metric on $\tilde{M}_{i}$, converges to the metric $\hat{g}_{\infty}$ of $\tilde{X}$ on compact subsets.

For simplicity, we identify $\mathbf{R}^{n}$ with the vector space $\left(T_{x} \tilde{X}, \hat{g}_{\infty}\right)$. We fix a positive number $R$ and a large $i_{0}$ with $i_{0}>R$. Let $\exp _{x}^{i}: B\left(2 i_{0}\right) \rightarrow$ $B_{3 i_{0}}(x, \tilde{X})$ be the $\tilde{g}_{i}$-exponential map. Passing to a subsequence if necessary, we may assume that the sequence $\left(B\left(2 i_{0}\right), h_{i}\right), h_{i}=\exp _{x}^{i *}\left(\hat{g}_{i}\right)$, converges to a $C^{1, \alpha}$ Riemannian $n$-manifold for the Hausdorff distance. 
It should be remarked that $h_{i}$ does not converge in general. Hence we use the center of mass technique again to obtain an imbedding $\psi_{i}^{*}: B\left(i_{0}\right) \rightarrow$ $B\left(2 i_{0}\right)$, so that the induced metric $\hat{h}_{i}=\psi_{i}^{*} h_{i}$ converges to a $C^{1, \alpha}$ metric $\hat{h}_{\infty}$ on $B\left(i_{0}\right)$. From the center of mass construction, $\psi_{i}$ can be chosen so as to satisfy the following conditions:

(6.3.1) $e^{-\delta\left(i_{0}\right)}|V|_{h_{i_{0}}}<\left|d \psi_{i}(v)\right|_{h_{i}}<e^{\delta\left(i_{0}\right)}|v|_{h_{i_{0}}}$ for every tangent vector $v$, where $\lim _{i_{0} \rightarrow \infty} \delta\left(i_{0}\right)=0$.

(6.3.2) $\psi\left(B\left(i_{0}\right)\right)$ includes $B(R)$.

If we set $F_{i}=\exp _{x}^{i} \psi_{i}$, we have the following diagram:

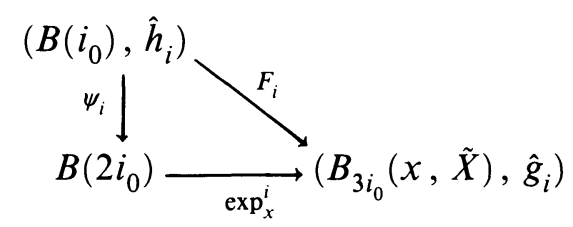

Sublemma 6.4. The map $\psi_{i}$ uniformly converges to a homeomorphism $\psi_{\infty}$ of $B\left(i_{0}\right)$ into $B\left(2 i_{0}\right)$ satisfying $\psi_{\infty}\left(B\left(i_{0}\right)\right) \supset B(R)$.

Proof. By Rauch's comparison theorem and the convergence $\hat{g}_{i} \rightarrow \hat{g}_{\infty}$, we have

$$
C\left(i_{0}\right)^{-1}|w|_{e}<|w|_{h_{i}}<C\left(i_{0}\right)|w|_{e}
$$

for every tangent vector $w$ to $B\left(2 i_{0}\right)$, where ||$_{e}$ is the norm induced by the inner product $\hat{g}_{\infty}$ on $\mathbf{R}^{n}$. Hence $(6.3 .1)$ yields

$$
C^{-1}|v|_{h_{i_{0}}}<\left|d \psi_{i}(v)\right|_{e}<C|v|_{h_{i_{0}}}
$$

for a uniform constant $C=C\left(i_{0}\right)$. It follows from Ascoli-Alzera's Theorem that $\psi_{i}$ uniformly converges to a bi-Lipschitz homeomorphism $\psi_{\infty}$. Property (6.3.2) of $\psi_{i}$ passes to the limit.

If we set $F_{\infty}=\exp _{x}^{\infty} \psi_{\infty}$, we have the following diagram:

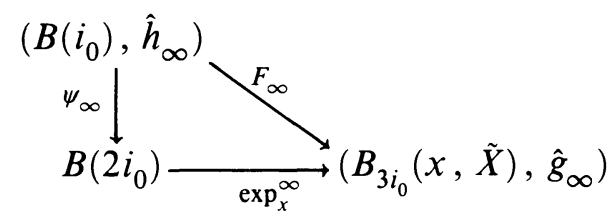

Sublemma 6.5. The map $F_{\infty}$ carries $\hat{h}_{\infty}$-geodesics to $\hat{g}_{\infty}$-geodesics in the length-preserving way.

Proof. For an $\hat{h}_{\infty}$-geodesic $\sigma$ in $B\left(i_{0}\right)$, take an $\hat{h}_{i}$-geodesic $\sigma_{i}$ so that $\lim \sigma_{i}=\sigma$. Since $F_{i}:\left(B\left(i_{0}\right), \hat{h}_{i}\right) \rightarrow\left(B_{3 i}(x, \tilde{X}), \hat{g}_{i}\right)$ is a local isometry, $F_{i} \sigma_{i}$ is a $\hat{g}_{i}$-geodesic. By taking a subsequence if necessary, we may assume 
that $F_{i} \sigma_{i}$ converges to a $\hat{g}_{\infty}$-geodesic $\tau$. Thus by the uniform convergence of $F_{i}$ to $F_{\infty}$, we have $\tau=F_{\infty}(\sigma)$, and

$$
\begin{gathered}
\text { length } \tau=\lim \text { length } F_{i} \sigma_{i}=\lim \text { length } \sigma_{i}=\text { length } \sigma, \\
\text { length } \tau=\lim \text { length } F_{i} \sigma_{i}=\lim \text { length } \sigma_{i}=\text { length } \sigma .
\end{gathered}
$$

Proof of Lemma 6.2. For any $u$ in $B\left(i_{0}\right)$, put $z=F_{\infty}(u)$. Take a small $\delta>0$ so that the ball $B_{\delta}\left(z, X_{0}\right)$ is convex. Then Sublemma 6.5 implies that $F_{\infty}$ maps $B_{\delta}\left(u, \hat{h}_{\infty}\right)$ onto $B_{\delta}\left(z, X_{0}\right)$ homeomorphically. Together with Sublemma 6.4 , this implies that $\exp _{x}^{\infty}$ is a local homeomorphism, because $R$ is arbitrary. Since $\exp _{x}^{\infty}$ is surjective and $\tilde{X}$ is simply connected, this yields the injectivity of $\exp _{x}^{\infty}$ by a standard covering argument.

Sublemma 6.6. The injectivity radius of $M_{i}$ goes to infinity as $i \rightarrow \infty$.

Proof. Suppose the contrary. Then, by the curvature assumption, for large $i$, there is a geodesic loop $\gamma_{i}$ in $\tilde{M}_{i}$ with uniformly bounded length. Since $d\left(M_{i}\right)<D$, we may assume that the distance between $y_{i}$ and the base point of the geodesic loop is less than a constant independent of $i$. Hence, the $\hat{g}_{i}$-geodesic $\varphi_{i}^{-1}\left(\gamma_{i}\right)$ converges to a $\hat{g}_{\infty}$-geodesic loop. This contradicts Lemma 6.2.

Lemma 6.1 is an immediate consequence of Sublemma 6.6.

\section{Covering spaces along fibers}

In this and the next sections, we shall prove Theorem 0.2 . Let $M_{i}$ be a sequence of Riemannian manifolds such that $M_{i} \in \mathscr{M}(n, 1), K_{M_{i}}<1 / i$, and let $X / \Gamma$ be the limit of $M_{i}$. It suffices to show that, for each sufficiently large $i$, there exists $\pi_{i}: M_{i} \rightarrow X / \Gamma$ satisfying $(0.3 .1)-(0.3 .4)$. In $\S \S 1-4$, we have constructed $\pi_{i}$ satisfying $(0.3 .1)$ and (0.3.2). [10] implies that this map satisfies (0.3.3). Let $H_{i} / \Lambda_{i}$ denote the fibers. We take the upper central series $\Lambda_{i}^{(0)} \subset \cdots \subset \Lambda_{i}^{\left(N_{i}\right)}$ of $\Lambda_{i} \cap H_{i}$. In other words, $\Lambda_{i}^{(j+1)} / \Lambda_{i}^{(j)}$ is the center of $\Lambda_{i}^{\left(N_{i}\right)} / \Lambda_{i}^{(j)}=\left(\Lambda_{i} \cap H_{i}\right) / \Lambda_{i}^{(j)}$. We shall refine this series so that $(0.3 .4)$ is satisfied. Fix $m$, and consider $m$ th component $J_{i, m}: \pi_{1}\left(M_{i}\right) \rightarrow \operatorname{Aut}\left(\Lambda_{i}^{(m)} / \Lambda_{i}^{(m-1)}\right)=\operatorname{SL}\left(k_{i}^{(m)}, \mathbf{Z}\right)$. Hereafter we shall omit the symbol $m$ and write $J_{i}, \hat{\Lambda}_{i}$, and $\bar{\Lambda}_{i}$ in place of $J_{i, m}, \Lambda_{i}^{(m)}$, and $\Lambda_{i}^{(m-1)}$. By taking a subsequence if necessary, we may assume that $k_{i}^{(m)}$ does not depend on $i$. We put $k=k_{i}^{(m)}$. Let $\bar{k}$ denote the rank of the nilpotent group $\left(\Lambda_{i} \cap H_{i}\right) / \Lambda_{i}^{(m)}$, which, we assume, is also independent of $i$. In this section, we shall prove the following. 
Lemma 7.1. There exist subgroups $\Lambda_{i, j}, \Lambda_{i, j}^{\prime}, j=0, \cdots, N$, of $\hat{\Lambda}_{i}$, Riemannian manifolds $Y_{j}$, and positive integers $s_{j}$, such that the following holds (by $M_{i, j}$ and $M_{i, j}^{\prime}$, we denote the covering spaces of $M_{i}$ corresponding to $\Lambda_{i, j}$ and $\Lambda_{i, j}^{\prime}$, respectively):

(7.2.1) $\Lambda_{i, 0}=\hat{\Lambda}_{i}, \Lambda_{i, N}=\bar{\Lambda}_{i}, \Lambda_{i, j+1} \subset \Lambda_{i, j}^{\prime} \subset \Lambda_{i, j}$.

(7.2.2) $Y_{j}$ is isometric to the direct product of $X \times \mathbf{R}^{\bar{k}+s_{0}+\cdots+s_{j-1}}$ and an $s_{j}$-dimensional flat torus.

(7.2.3) $\Lambda_{i, j}$ is a normal subgroup of $\pi_{1}\left(M_{i}\right)$.

(7.2.4) $\Lambda_{i, j} / \Lambda_{i, j+1} \simeq \mathbf{Z}^{s_{j}}$.

(7.2.5) $\left[\Lambda_{i, j}: \Lambda_{i, j}^{\prime}\right]<\infty$. Furthermore $\Lambda_{i, j}^{\prime} / \Lambda_{i, j+1}=l \cdot \mathbf{Z}^{s_{j}} \subset \mathbf{Z}^{s_{j}}=$ $\Lambda_{i, j} / \Lambda_{i, j+1}$ for some number $l$ depending on $i$ and $j$.

(7.2.6) We can find $p_{i, j} \in M_{i, j}$, such that $\left(M_{i, j}, p_{i, j}\right)$ converges to the universal covering space $X \times \mathbf{R}^{\bar{k}+s_{0}+\cdots+s_{j-1}}$ of $Y_{j-1}$, with respect to the pointed Hausdorff distance.

(7.2.7) We can find $p_{i, j}^{\prime}$ so that $M_{i, j}^{\prime}$ converges to $Y_{j}$ with respect to the pointed Hausdorff distance.

Proof. Let $M_{i, 0}$ be the covering space of $M_{i}$ corresponding to $\hat{\Lambda}_{i}$. Then, in view of Lemma 6.1, we can prove the following.

Sublemma 7.3. We can take $p_{i, 0} \in M_{i, 0}$ such that $\left(M_{i, 0}, p_{i, 0}\right)$ converges to $\left(X \times \mathbf{R}^{\bar{k}}, p_{\infty, 0}\right)$ with respect to the pointed Hausdorff distance.

Therefore, by [7], there exists a submersion $\hat{\pi}_{i, 0}: B_{2}\left(p_{i, 0}, M_{i, 0}\right) \rightarrow X \times$ $\mathbf{R}^{\bar{k}}$ for each sufficiently large $i$. Set $\bar{M}_{i, 0}=\left(\hat{\pi}_{i, 0}\right)^{-1}\left(B_{1}\left(p_{\infty, 0}, X \times \mathbf{R}^{\bar{k}}\right)\right)$. By restricting $\hat{\pi}_{i, 0}$, we obtain a fibration $\bar{\pi}_{i, 0}: \bar{M}_{i, 0} \rightarrow B_{1}\left(p_{\infty, 0}, X \times \mathbf{R}^{\bar{k}}\right)$. We put $\tilde{Y}_{0}=X \times \mathbf{R}^{\bar{k}}$ and $\tilde{Y}_{0, c}=B_{1}\left(p_{\infty, 0}, X \times \mathbf{R}^{\bar{k}}\right)$. Then, we can prove

Sublemma 7.4. $\pi_{1}\left(\bar{M}_{i, 0}\right)=\hat{\Lambda}_{i}$.

Let $\Lambda_{i, 0}(l)$ be the subgroup satisfying $\Lambda_{i, 0}(l) / \bar{\Lambda}_{i}=l \cdot \mathbf{Z}^{k} \subset \mathbf{Z}^{k}=$ $\hat{\Lambda}_{i} / \bar{\Lambda}_{i}$, and denote by $\bar{M}_{i, 0}(l)$ the covering space of $\bar{M}_{i, 0}$ corresponding to $\Lambda_{i, 0}(l)$. Then we have a fibration $\bar{\pi}_{i, 0}(l): \bar{M}_{i, 0}(l) \rightarrow \tilde{Y}_{0, c}$. Put

$$
\alpha_{i, l}=\operatorname{diam}\left(\left(\bar{\pi}_{i, 0}(l)\right)^{-1}\left(p_{\infty, 0}\right)\right)
$$

Then

(7.5.1) $\quad \alpha_{i, l+1} / \alpha_{i, l}<C$ for some constant $C$ independent of $i$,

$$
\lim _{l \rightarrow \infty} \alpha_{i, l}=\infty
$$


Hence, we can choose $l_{i}$ so that $1<\alpha_{i, l_{i}}<C$. Put $\Lambda_{i, 0}^{\prime}=\Lambda_{i, 0}\left(l_{i}\right)$, and let $M_{i, 0}^{\prime}$ and $\bar{M}_{i, 0}^{\prime}$ denote the covering spaces of $M_{i}$ and $\bar{M}_{i, 0}$ corresponding to $\Lambda_{i, 0}^{\prime}$, respectively. In other words $\bar{M}_{i, 0}^{\prime}=M_{i, 0}\left(l_{i}\right)$. Let $\bar{\pi}_{i, 0}^{\prime}=\pi_{i, 0}\left(l_{i}\right): \bar{M}_{i, 0}^{\prime} \rightarrow \tilde{Y}_{0, c}$. Since

$$
1<\operatorname{diam}\left(\left(\bar{\pi}_{i, 0}^{\prime}\right)^{-1}\left(p_{\infty, 0}\right)\right)<C,
$$

and $\bar{\pi}_{i, 0}^{\prime}$ is an almost Riemannian submersion, we have $\operatorname{diam}\left(\bar{M}_{i, 0}^{\prime}\right)<$ $2(4+C)$. Therefore, by taking a subsequence if necessary, we may assume that $\bar{M}_{i, 0}^{\prime}$ converges to a metric space $\bar{Y}_{1}$ with respect to the Hausdorff distance. We fix a point $p_{i, 0}^{\prime}$ on $\left(\bar{\pi}_{i, 0}^{\prime}\right)^{-1}\left(p_{\infty, 0}\right)$. We may assume that $\left(M_{i, 0}^{\prime}, p_{i, 0}^{\prime}\right)$ converges to a metric space $\left(Y_{1}, p^{(1)}\right)$ with respect to the pointed Hausdorff distance. Remark $Y_{1} \supset \bar{Y}_{1}$. Let $G$ be as in $\S 2$. Then, by construction, there exists a closed subgroup $G_{1}$ of $G$ such that $Y_{1}$ is isometric to $X / G_{1}$. By $\S \S 3$ and $4, Y_{1}$ is therefore a nonpositively curved orbifold of $C^{1, \alpha}$-class. Using [7], we thus have the following.

Sublemma 7.7. After perturbing $\bar{Y}_{1} \subset Y_{1}$ and $\bar{M}_{i, 0}^{\prime} \subset M_{i, 0}^{\prime}$ in a small neighborhood of their boundaries, we can construct fibrations $\hat{\pi}_{i, 0}^{\prime}: \bar{M}_{i, 0}^{\prime} \rightarrow$ $\bar{Y}_{1}$ and $P_{1}: \bar{Y}_{1} \rightarrow \tilde{Y}_{1, c}$, such that $\bar{\pi}_{i, 0}^{\prime}=P_{1} \hat{\pi}_{i, 0}^{\prime}$.

Sublemma 7.8. The inclusion $\bar{Y}_{1} \rightarrow Y_{1}$ induces isomorphisms on fundamental groups.

Sublemma 7.7 implies that $\pi_{1}\left(\bar{Y}_{1}\right)$ is nilpotent, so that so is $\pi_{1}\left(Y_{1}\right)$. Since $Y_{1}$ is a nonpositively curved Riemannian orbifold and every element of $\pi_{1}\left(Y_{1}\right)$ is represented by a closed geodesic, Lemma 3.8 implies

Sublemma 7.9. $\pi_{1}\left(Y_{1}\right) \simeq \mathbf{Z}^{s_{0}}$. Furthermore $Y_{1}$ is isometric to the direct product of $X \times \mathbf{R}^{\bar{k}}$ and an $s_{0}$-dimensional flat torus.

Let $\Lambda_{i}$ i $=\pi_{1}\left(\left(\hat{\pi}_{i, 0}^{\prime}\right)^{-1}\left(p^{(1)}\right)\right)$. Then we have an exact sequence

$$
1 \rightarrow \Lambda_{i, 1} \rightarrow \Lambda_{i, 0}^{\prime} \rightarrow \mathbf{Z}^{s_{0}} \rightarrow 1 \text {. }
$$

(Recall that $\Lambda_{i, 0}^{\prime}=\pi_{1}\left(\bar{M}_{i, 0}^{\prime}\right)$ and $\mathbf{Z}^{s_{0}}=\pi_{1}\left(\bar{Y}_{1}\right)$, and that (7.10) is the homotopy exact sequence associated to the fibration $\left.\hat{\pi}_{i, 0}.\right)$ Remark that $\Lambda_{i, 1} \supset \bar{\Lambda}_{i}$. If $\Lambda_{i, 1}=\bar{\Lambda}_{i}$ (or equivalently $s_{0}=k$ ), we finish the construction. If not, we will continue by proving the following sublemma. By $M_{i, 1}$, we denote the covering space of $M_{i}$ corresponding to $\Lambda_{i, 1}$. 
Sublemma 7.11. We can find $p_{i, 1} \in M_{i, 1}$ such that $\left(M_{i, 1}, p_{i, 1}\right)$ converges to $\left(X \times \mathbf{R}^{\bar{k}+s_{0}}, p_{\infty, 1}\right)$ with respect to the pointed Hausdorff distance.

Using Sublemma 7.11 in place of Sublemma 7.3, we obtain $\bar{M}_{i, 1} \subset$ $M_{i, 1}$ and a fibration $\bar{\pi}_{i, 1}: \bar{M}_{i, 1} \rightarrow \tilde{Y}_{1, c}$. Define $\Lambda_{i, 1}(l)$ by $\Lambda_{i, 1}(l) / \bar{\Lambda}_{i}=$ $l \cdot \mathbf{Z}^{k-s_{1}} \subset \mathbf{Z}^{k-s_{1}}=\Lambda_{i, 1} / \bar{\Lambda}_{i}$, and denote by $M_{i, 1}(l)$ and $\bar{M}_{i, 1}(l)$ the covering spaces of $M_{i, 1}$ and $\bar{M}_{i, 1}$ corresponding to $\Lambda_{i, 1}(l)$. We take $l_{i, 1}$ satisfying a condition similar to (7.6). Let $\Lambda_{i, 1}^{\prime}=\Lambda_{i, 1}\left(l_{i, 1}\right), M_{i, 1}^{\prime}=$ $M_{i, 1}\left(l_{i, 1}\right)$, and $\bar{M}_{i, 1}^{\prime}=\bar{M}_{i, 1}\left(l_{i, 1}\right)$, and define the fibration $\bar{\pi}_{i, 1}^{\prime}: \bar{M}_{i, 1}^{\prime} \rightarrow$ $\tilde{Y}_{1, c}$ similarly. Choose $p_{i, 1}^{\prime} \in\left(\bar{\pi}_{i, 1}^{\prime}\right)^{-1}\left(p_{\infty, 1}\right) \subset \bar{M}_{i, 1}^{\prime} \subset M_{i, 1}^{\prime}$. Then, by taking a subsequence if necessary, we may assume that $\bar{M}_{i, 1}^{\prime}$ converges to a space $\bar{Y}_{2}$ with respect to the Hausdorff distance and that $\left(M_{i, 1}^{\prime}, p_{i, 1}^{\prime}\right)$ converges to $\left(Y_{2}, p^{(2)}\right)$ with respect to the pointed Hausdorff distance. Therefore, by proving sublemmas similar to 7.7-7.9, we can find $\Lambda_{i, 2}$ and $s_{1}$. Repeating this, we obtain $\Lambda_{i, j}, \Lambda_{i, j}^{\prime}, s_{j}$ and $Y_{j}$. Remark that $1<\alpha_{i, l_{i}}$ implies $s_{0}>0$. Similarly $s_{j}>0$. Hence our construction stops after finitely many repetitions. We omit the verification of properties (7.2.1)-(7.2.7). The proof of Lemma 7.1 is now complete.

\section{Finiteness of $J\left(\pi_{1}\left(M_{i}\right)\right)$}

Property (7.2.3) implies that the map $J_{i}: \pi_{1}\left(M_{i}\right) \rightarrow \operatorname{SL}(k ; Z)$ induces a homomorphism $J_{i}^{\prime}: \pi_{1}\left(M_{i}\right) \rightarrow \prod \mathrm{SL}\left(s_{j} ; \mathbf{Z}\right)$. (Remark that $\sum s_{j}=$ $k$.) Let $J_{i, j}: \pi_{1}\left(M_{i}\right) \rightarrow \mathrm{SL}\left(s_{j} ; \mathbf{Z}\right)$ be the $j$ th component, and $\gamma$ be an arbitrary element of $\Gamma$. Choose elements $\gamma_{i} \in \pi_{1}\left(M_{i}\right)$ such that $\left(\pi_{i}\right)_{*}\left(\gamma_{i}\right)=\gamma$. It suffices to show that $J_{i, j}\left(\gamma_{i}\right)$ is of finite order for sufficiently large $i$. Let $\Lambda_{i, j}(\gamma)$ and $\Lambda_{i, j}^{\prime}(\gamma)$ denote the subgroups generated by $\gamma_{i}$ and $\Lambda_{i, j}, \Lambda_{i, j}^{\prime}$, respectively. The covering spaces of $M_{i}$ corresponding to $\Lambda_{i, j}(\gamma)$ and $\Lambda_{i, j}^{\prime}(\gamma)$ are denoted by $M_{i, j}(\gamma)$ and $M_{i, j}^{\prime}(\gamma)$, respectively. By definition, $\mathbf{Z}$ acts on $M_{i, j}$ and $M_{i, j}^{\prime}$ by isometry such that the quotient spaces $M_{i, j} / \mathbf{Z}$ and $M_{i, j}^{\prime} / \mathbf{Z}$ are isometric to $M_{i, j}(\gamma)$ and $M_{i, j}^{\prime}(\gamma)$, respectively. We can find a $Z$-action on $Y_{j}$ such that $\left(M_{i, j}^{\prime}, \mathbf{Z}, p_{i, j}^{\prime}\right)$ converges to $\left(Y_{j}, \mathbf{Z}\right)$ with respect to the $\mathbf{Z}$-pointed Hausdorff distance (see $[8,1.12])$. We put $Y_{j}(\gamma)=Y_{j} / \mathbf{Z}$. We can replace $Y_{j}(\gamma)$ by its homotopy equivalent compact subset, and construct a homomorphism $\Lambda_{i, j}^{\prime}(\gamma)=\pi_{1}\left(M_{i, j}^{\prime}\right) \rightarrow \pi_{1}\left(Y_{j}(\gamma)\right)$ such that the following diagram is 
commutative and exact:

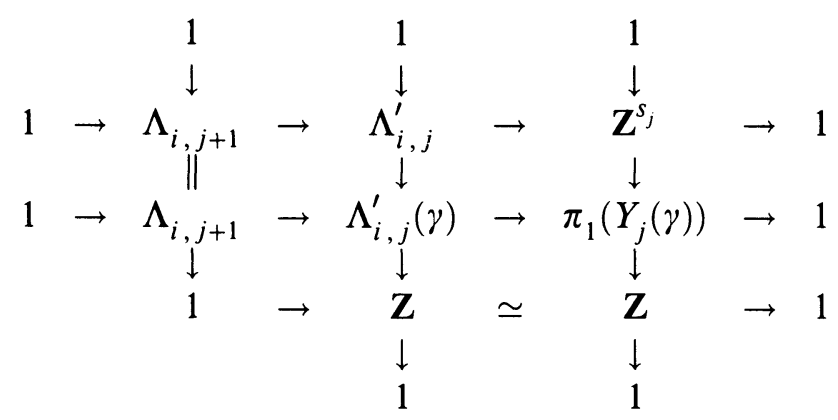

Now, we consider the following commutative diagram:

$$
\begin{aligned}
& 1 \rightarrow \mathbf{Z}^{s_{j}} \rightarrow \pi_{1}\left(Y_{j}(\gamma)\right) \rightarrow \mathbf{Z} \rightarrow 1
\end{aligned}
$$

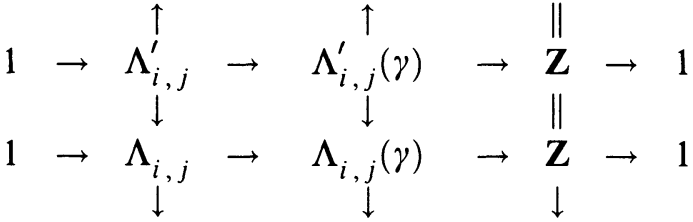

$$
\begin{aligned}
& 1 \rightarrow \Lambda_{i} \rightarrow \pi_{1}\left(M_{i}\right) \rightarrow \Gamma \rightarrow 1
\end{aligned}
$$

It follows from the commutativity of the diagram that $J_{i, j}\left(\gamma_{i}\right)$ coincides with the action of $1 \in \mathbf{Z}$ on $\mathbf{Z}^{s_{j}}$ induced from the exact sequence

$$
1 \rightarrow \mathbf{Z}^{s_{j}} \rightarrow \pi_{1}\left(Y_{j}(\gamma)\right) \rightarrow \mathbf{Z} \rightarrow 1
$$

On the other hand, since $Y_{j}(\gamma)$ is complete and of nonpositive curvature, and every element of $\pi_{1}\left(Y_{j}(\gamma)\right)$ is represented by a closed geodesic, it follows from Lemma 3.8 that $Y_{j}(\gamma)$ has a finite covering $\tilde{Y}_{j}(\gamma)$ such that $\pi_{1}\left(\tilde{Y}_{j}(\gamma)\right) \simeq \mathbf{Z}^{s_{j}} \times \mathbf{Z}$. Consequently $J_{i, j}(\gamma)$ is of finite order. This completes the proof of Theorem 0.2 .

\section{Construction of almost nonpositively curved metrics}

In this section, we shall prove Theorem 0.4. First we assume that $X / \Gamma=N$ is a smooth Riemannian manifold. Our construction is a modification of one in $[10, \S 6]$, but is not exactly the same because we need a metric satsifying more restrictive curvature assumptions than those in [10, §6]. We have a fibration $H / \Lambda \rightarrow M \rightarrow N$ whose structure group is contained in $T=C(H) /(C(H) \cap \Lambda) \tilde{x} L$, where $L$ is a group satisfying (0.3.4). We have a $T$-connection of $\pi$, which gives a decomposition of $T_{x}(M)$ to its horizontal subspace $H_{x}(M)$ and its vertical subspace 
$V_{x}(M)=T_{x}\left(\pi^{-1} \pi(x)\right)$. Put

$$
\begin{gathered}
g_{\varepsilon}(V, W)=g_{N}\left(\pi_{*}(V), \pi_{*}(W)\right) \quad \text { if } V, W \in H_{x}(M), \\
g_{\varepsilon}(V, W)=0 \text { if } V \in H_{x}(M) \text { and } W \in V_{x}(M) .
\end{gathered}
$$

We shall define $g_{\varepsilon}(V, W)$ for $V, W \in V_{x}(M)$. Let $\pi_{1}: P_{1} \rightarrow N$ be the principal $T$-bundle associated to $\pi$, and let $\pi_{2}: P_{2} \rightarrow N$ be the principal $L$-bundle induced from $P_{1}$. (In other words, $P_{2}=P_{1} /(C(H) / C(H) \cap \Lambda)$ ). Let $\Lambda^{(m)}$ be as in (0.3.4), and denote by $H_{m}$ the Zariski closure of $\Lambda^{(m)}$ in $H$. Let $\mathfrak{h}$ and $\mathfrak{h}_{m}$ be the Lie algebras of $H$ and $H_{m}$, respectively. Since $\Lambda^{(m)}$ is $L$-invariant, so is $\mathfrak{h}_{m}$. Hence, we can define vector bundles $\mathscr{H}$ and $\mathscr{H}_{m}$ by $\mathscr{H}=P_{2} \times_{L} \mathfrak{h}$ and $\mathscr{H}^{(m)}=P_{2} \times_{L} \mathfrak{h}_{m}$. We put $\mathscr{L}^{(m)}=\mathscr{H}^{(m)} / \mathscr{H}^{(m-1)}$. Since $L$ is discrete, our vector bundles $\mathscr{H}, \mathscr{H}^{(m)}$, and $\mathscr{L}$ are flat. Furthermore, (0.3.4) implies that the holonomy group of the flat bundle $\mathscr{L}^{(m)}$ is finite. Hence there exists a metric $h_{m}$ on $\mathscr{L}^{(m)}$ compatible with its flat structure. In other words, for each small open set $U$ of $N$, there exist sections $a_{1}, \cdots, a_{k_{m}}$ of $\mathscr{L}^{(m)}$ such that $h_{m}\left(a_{i}, a_{j}\right)=\delta_{i, j}$ and $\nabla a_{i}=0$, where $\nabla$ is the covariant derivative associated to the flat structure of $\mathscr{L}^{(m)}$, the symbol $\delta_{i, j}$ is Kroneker's delta, and $k_{m}=\operatorname{rank} \mathscr{L}^{(m)}$. We define a metric $h$ on $\mathscr{H}$ so that the metric induced on $\mathscr{L}^{(m)}$ from $h$ is equal to $h_{m}$. Then, we can easily prove the following lemma. We set $o^{\prime}(i)=m$ if $k_{m-1}<i \leq k_{m}$. Put $k=\operatorname{rank} \mathscr{H}$.

Lemma 9.2. Let $p \in N$, and let $U$ be a neighborhood of $p$. Then we can find $C^{\infty}$-sections $v_{1}, v_{2}, \cdots, v_{k}, v_{1}^{\prime}, \cdots, v_{k}^{\prime}$, of $\mathscr{H}$ such that (9.3.1) $\nabla v_{i}^{\prime}=0$, where $\nabla$ is the connection associated to the flat structure of $\mathscr{H}$,

(9.3.2) $\left(v_{1}, \cdots, v_{k}\right)$ is an orthonormal base of $\mathscr{H}$ at each point of $U$,

(9.3.3) $v_{i}, v_{i}^{\prime} \in \mathscr{H}^{\left(o^{\prime}(i)\right)}$,

(9.3.4) $v_{i}-v_{i}^{\prime} \in \mathscr{H}^{\left(o^{\prime}(i)-1\right)}$.

Now, we fix $p$ and $U$. Let $\varphi: \pi^{-1}(U) \rightarrow U \times H / \Lambda$ be a local trivialization of $\pi$. Since $P_{2}$ is a fiber bundle induced from the associate principal bundle $P_{1}$ of $\pi$, it follows that $\varphi$ determines a local trivialization $\varphi^{\prime}:\left.\mathscr{H}\right|_{U} \rightarrow U \times \mathfrak{h}$ of $\mathscr{H}$. The trivialization $\varphi^{\prime}$ and the metric $h$ determine a quadratic form $h_{x}$ on $\mathfrak{h}$ for each $x \in U$. Formula (9.3.1) implies that the second component of $\varphi^{\prime}\left(v_{i}^{\prime}(x)\right.$ ) (which is contained in $\mathfrak{h}$ ) does not depend on $x$. We put $\varphi^{\prime}\left(v_{i}(x)\right)=\left(x, \tilde{v}_{i}(x)\right)$. Then by Lemma 
9.2 we have

$$
\begin{gathered}
\varphi^{\prime}\left(v_{i}\right)=\left(x, \tilde{v}_{i}(x)\right), \\
\varphi^{\prime}\left(v_{i}^{\prime}\right)=\left(x, \tilde{v}_{i}^{\prime}\right), \\
\tilde{v}_{i}^{\prime}-\tilde{v}_{i}(x) \in \mathfrak{h}_{o^{\prime}(i)-1} .
\end{gathered}
$$

Putting $o(i)=o^{\prime}(k)-o^{\prime}(i)$, we define a quadratic form $h_{x, \varepsilon}$ on $\mathfrak{h}$ by

$$
h_{x, \varepsilon}\left(v_{i}^{\prime}(x), v_{i}^{\prime}(x)\right)=\delta_{i, j} \cdot \varepsilon^{3^{o(i)}} .
$$

Since $\Lambda /(H \cap \Lambda)$ is finite, we may assume that $h_{x}$ is $\Lambda /(H \cap \Lambda)(\subset \operatorname{Aut}(\Lambda))$ invariant, so that so is $h_{x, \varepsilon}$. Therefore, $h_{x, \varepsilon}$ induces a Riemannian metric on $H / \Lambda$. Using this metric on the vertical direction and using (9.1.1) and (9.1.2) on other directions, we obtain a Riemannian metric on $U \times H / \Lambda \simeq \pi^{-1}(U)$. It is straightforward to show that these metrics can be patched together to give a metric $g_{\varepsilon}$ on $M$. Clearly $g_{\varepsilon}$ satisfies $(0.5 .2)$ and (0.5.3). Thus we need only to show (0.5.1). To see this, since the problem is local, it suffices to estimate the curvature on $U \times H$. By $e_{1}^{\prime}, \cdots, e_{l}^{\prime}$, we denote the orthonormal frame of the tangent bundle on $U$, and by $e_{1}, \cdots, e_{l}$ we denote their horizontal lifts to $U \times H$. The elements $v_{i}(x)$ define vector fields on $\{x\} \times H$. Hence we get vector fields $f_{i}$ on $U \times H$. The element $\tilde{v}_{i}^{\prime}$ of $\mathfrak{h}$ induces a vector field $f_{i}^{\prime}$ on $U \times H$. By definition, $\left(e_{1}, \cdots, e_{l}, f_{1}, \cdots, f_{k}\right)$ is an orthonormal frame of the Riemannian manifold $\left(U \times H, g_{1}\right)$, and $\left(e_{1}, \cdots, e_{l}, \varepsilon^{-3^{o(1)}} \cdot f_{1}, \cdots, \varepsilon^{-3^{o(k)}} \cdot f_{k}\right)$ is one of $\left(U \times H, g_{\varepsilon}\right)$. We shall calculate the commutators of $e_{i}$ and $f_{i}$. Since $\pi$ is a $T$-connection, it follows that

$$
\left[e_{i}, e_{j}\right]=\sum_{q=1}^{l} a_{i, j}^{q} \cdot e_{q}+\sum_{q=1}^{k} b_{i, j}^{q} \cdot f_{q},
$$

where $a_{i, j}^{q}$ and $b_{i, j}^{q}$ are functions on $U$. (In other words, they do not depend on $H$ factors.) Since $\left[\mathfrak{h}, \mathfrak{h}_{k}\right] \subset \mathfrak{h}_{k-1}$, we have

$$
\left[f_{i}, f_{j}\right]=\sum_{\substack{o(q)>o(i) \\ o(q)>o(j)}} c_{i, j}^{q} \cdot f_{q},
$$

where $c_{i, j}^{q}$ is a function on $U$. We shall calulate $\left[e_{i}, f_{j}\right]$. First remark that $f_{j}^{\prime}$ is a vector field generated by an element of $\mathfrak{h}$. On the other hand, since our connection of $\pi$ is a $T$-connection, it follows that the horizontal lifts $e_{i}$ are $H$-invariant. Therefore

$$
\left[e_{i}, f_{j}^{\prime}\right]=0 .
$$


Second, (9.4.3) implies that there exists a function $\alpha_{i, j}$ on $U$ such that

$$
f_{i}=f_{i}^{\prime}+\sum_{o(j)>o(i)} \alpha_{i, j} \cdot f_{j}^{\prime} .
$$

We can regard $U$ as an open subset of $\mathbf{R}^{n}$. We put

$$
e_{i}^{\prime}=\sum_{j=1}^{l} \beta_{i, j} \cdot \frac{\partial}{\partial x^{j}} .
$$

Hence, by (9.7.1) and (9.7.2) we have

$$
\left[e_{i}, f_{j}\right]=\sum_{\substack{o(q)>o(j) \\ l=1, \cdots, n}} \beta_{i, l} \cdot \frac{\partial \alpha_{j, q}}{\partial x^{l}} \cdot f_{q}^{\prime} .
$$

Consequently, we have

$$
\left[e_{i}, f_{j}\right]=\sum_{o(q)>o(j)} d_{i, j}^{q} \cdot f_{q},
$$

where $d_{i, j}^{q}$ is a function on $U$. (Compare [10, formula (6.4.3)], where the right-hand side was $\sum_{o(q) \geq o(j)} d_{i, j}^{q} \cdot f_{q}$.) Now, we put $f_{i, \varepsilon}=\varepsilon^{-3^{o(i)}} f_{i}$. Let $\left(e^{1}, e^{2}, \cdots, e^{n}, f_{\varepsilon}^{1}, f_{\varepsilon}^{2}, \cdots, f_{\varepsilon}^{k}\right)$ be the dual base to $\left(e_{1}, \cdots, e_{n}, f_{1, \varepsilon}\right.$, $\left.\cdots, f_{k, \varepsilon}\right)$. Formulas (9.6.1), (9.6.2), and (9.7.3) imply

$$
\begin{gathered}
d e^{i}=\sum a_{j, q}^{i} \cdot e^{j} \wedge e^{q}, \\
d f_{\varepsilon}^{i}=\sum_{\substack{o(i)>o(j) \\
o(i)>o(q)}} c_{j, q}^{i} \cdot \varepsilon^{3^{o(i)}-3^{o(j)}-3^{o(q)}} \cdot f_{\varepsilon}^{j} \wedge f_{\varepsilon}^{q} \\
+\sum_{\substack{o(i)>o(q) \\
j=1, \cdots, n}} d_{j, q}^{i} \cdot \varepsilon^{3^{o(i)}-3^{o(q)}} e^{j} \wedge f_{\varepsilon}^{q} \\
+\sum b_{j, k}^{i} \cdot \varepsilon^{3^{o(i)}} e^{j} \wedge e^{q} .
\end{gathered}
$$

We remark that all the coefficients in formula (9.8.2) tend to 0 when $\varepsilon$ goes to 0 . On the other hand, we can calculate the curvatures of $\left(U \times H, g_{\varepsilon}\right)$ by symmetrizing the coefficients of (9.8.1) and (9.8.2). Hence, when $\varepsilon$ goes to 0 , the sectional curvatures of $g_{\varepsilon}$ tend to 0 except those coming from the symmetrization of $a_{i, j}^{q}$. Since $\left[e_{i}^{\prime}, e_{j}^{\prime}\right]=\sum a_{i, j}^{q} \cdot e_{q}^{\prime}$ and $e_{i}^{\prime}$, $i=1,2, \cdots$, is an orthonormal frame of the Riemannian manifold $U$, the symmetrization of $a_{i, j}^{q}$ gives the curvature of $U$. Inequality (0.5.3) follows immediately. 
Thus, we have verified Theorem 0.4 in the case when $X / \Gamma$ is nonsingular. We shall deal with the general case. Let $S \subset X / \Gamma$ be the set of the singular points. Vector bundles $\mathscr{H}, \mathscr{H}^{(m)}$, and $\mathscr{L}^{(m)}$ on $X / \Gamma-S$ can be constructed in a similar way. Let $p \in S$, and let $U$ be a neighborhood of $p$ in $X / \Gamma$. There exist a finite group $\Theta$ and a Riemannian manifold $V$ on which $\Theta$ acts by isometry such that $V / \Theta=U$. Let $P: V \rightarrow U$ be the natural projection. Vector bundles $\mathscr{H}, \mathscr{H}^{(m)}$, and $\mathscr{L}^{(m)}$ and the metric $h$ can be lifted to $\tilde{\mathscr{H}}, \tilde{\mathscr{H}}^{(m)}, \tilde{\mathscr{L}}^{(m)}$, and $\tilde{h}$ on $V-P^{-1}(S)$ respectively. By construction, they can be uniquely extended to $V$, which are denoted by the same symbols. We have a fibration $\tilde{\pi}: W \rightarrow V$ such that the following diagram commutes:

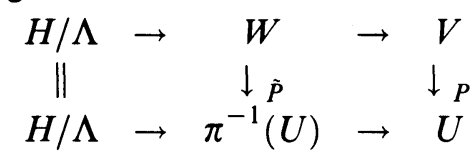

Here $\Theta$ acts on $W$ such that $W / \Theta \simeq V$, and $\tilde{P}$ is a covering map. Using $\mathscr{H}, \mathscr{H}^{(m)}, \mathscr{L}^{(m)}$, and $h$ in a similar way, we can construct Riemannian metrics on $W$. By construction, these metrics are $\Theta$-invariant. Hence they induce Riemannian metrics on $\pi^{-1}(U)$. It is straightforward to see that these metrics can be patched together to give Riemannian metrics satisfying $(0.5 .1),(0.5 .2)$, and $(0.5 .3)$. The proof of Theorem 0.4 is now complete:

Finally, we shall prove Corollary 0.7. By assumption and Theorem 0.2, the $C^{1, \alpha}$ metric on $X$ can be approximated by $\Gamma$-invariant $C^{\infty}$ metrics $\bar{g}_{\varepsilon}$ such that $-1<K\left(\bar{g}_{\varepsilon}\right)<\varepsilon$. This fact combined with Theorem 0.4 implies the conclusion.

\section{Aspherical manifolds with almost nonnegative curvature}

In this section, we shall prove Corollary 0.9. Let $M_{i}, i=1,2, \cdots$, be $K(\pi, 1)$-spaces in $\mathscr{M}(n, D)$ satisfying $-1 / i<K\left(M_{i}\right) \leq 1$. By taking a subsequence if necessary, we may assume that $M_{i}$ converges to a metric space with respect to the Hausdorff distance. As was discussed in $\S 6$, the universal covering $\tilde{M}_{i}$ of $M_{i}$ converges to a contractible $C^{1, \alpha}$ Riemannian $n$-manifold $\tilde{X}$ with respect to the pointed Hausdorff distance.

Lemma 10.1. $\quad \tilde{X}$ is isometric to the flat Euclidean space $\mathbf{R}^{n}$.

We assume the lemma for the moment. Let $G_{i}$ be the deck transformation group of the covering $\tilde{M}_{i} \rightarrow M_{i}$, and let $G$ be the limit of $G_{i}$, which is a subgroup of isometries of $\tilde{X}$. The argument in $\S \S 2$ and 3 shows that the identity component $G_{0}$ of $G$ is nilpotent and acts freely on $\tilde{X}$ (see 
also $[9, \S 4])$. Lemma 10.1 implies that $G_{0}$ consists of translations, and that the quotient $\tilde{X} / G$, the limit of $M_{i}$, is a flat orbifold. Lemma 10.1 also enables us to use the technique developed in $\S \S 7$ and 8 to construct a fibration $\pi_{i}: M_{i} \rightarrow \tilde{X} / G$ satisfying (0.3.1)-(0.3.4). Therefore, Theorem 0.4 shows that $M_{i}$ admits almost flat metrics. By [13], [21], $M_{i}$ is diffeomorphic to an infranilmanifold $H / \Lambda$.

To prove Lemma 10.1 , let $c:[0, \infty) \rightarrow \tilde{X}$ be a geodesic ray, and let $\delta$ be the Busemann function associated with $c: \delta(x)=\lim _{t \rightarrow \infty} t-$ $d(c(t), x)$. Although the metric of $\tilde{X}$ is only of class $C^{1, \alpha}$, using the curvature assumption $K\left(M_{i}\right)>-1 / i$, we can develop the basic construction of [4] on $\tilde{X}$ (see [4, Theorems 1.2 and 1.10]) to prove the following.

Sublemma 10.2. $\delta$ is convex.

Since $\tilde{X} / G$ is compact, there is a line $c$ in $\tilde{X}$. Let $\delta_{+}$and $\delta_{-}$be the Busemann functions associated with rays $\left.c\right|_{[0, \infty)}$ and $\left.c^{-1}\right|_{[0, \infty)}$ respectively. By the triangle inequality, $\delta_{+}+\delta_{-} \leq 0$. It follows from Sublemma 10.2 that $\delta_{+}=-\delta_{-}$is linear. Thus $\tilde{X}$ splits isometrically as $\tilde{X}=X_{1} \times \mathbf{R}$. Using the action of $G$, we can easily show that $X_{1}$ has a line. Repeating this process finitely many times completes the proof of Lemma 10.1 .

Remark 10.3. The authors are certain that Corollary 0.9 is still valid for manifolds of almost nonnegative Ricci curvature. The proof is not yet complete.

\section{References}

[1] W. Ballmann, M. Gromov \& V. Schroeder, Manifold of nonpositive curvature, Birkhauser, Boston, 1985.

[2] J. Bemelmans, Min-Oo, \& E. A. Ruh, Smoothing Riemannian metrics, Math. Z. 188 (1984) 69-74.

[3] P. Buser and D. Gromoll, On the almost negatively curved metrics on $S^{3}$, Geometry and Analysis on Manifolds (T. Sunada, ed.), Lecture Notes in Math., Vol. 1339, Springer, Berlin, 78-85.

[4] J. Cheeger \& D. Gromoll, On the structure of complete manifolds of nonnegative curvature, Ann. of Math. 96 (1972) 413-443.

[5] F. T. Farrell \& W. C. Hsiang, Topological characterization of flat and almost flat Riemannian manifolds $M^{n}(n \neq 3,4)$, Amer. J. Math. 105 (1983) 641-672.

[6] K. Fukaya, Theory of convergence for Riemannian orbifolds, Japanese J. Math. 12 (1986) 121-160.

[7] _ Collapsing Riemannian manifolds to ones of lower dimensions, J. Differential Geometry 25 (1987) 139-156.

[8] $\ldots$ boundary of the set of the Riemannian manifolds with bounded curvatures and diameters, J. Differential Geometry 28 (1988) 1-21.

[9] _ A compactness theorem of a set of aspherical Riemannian orbifolds, A Fete of Topology (Y. Matsumoto, T. Mizutani, and S. Morita, eds.), Academic Press, New York, 1988, 391-413. 
[10] _ Collapsing Riemannian manifolds to ones of lower dimension. II, J. Math. Soc. Japan 41 (1989) 333-356.

[11] R. E. Greene \& H. Wu, Lipschitz convergence of Riemannian manifolds, Pacific J. Math. 131 (1988) 119-141.

[12] D. Gromoll \& J. A. Wolf, Some relations between the metric structure and the algebraic structure of the fundamental group in manifolds of nonpositive curvature, Bull. Amer. Math. Soc. 77 (1971) 545-552.

[13] M. Gromov, Almost flat manifolds, J. Differential Geometry 13 (1978) 231-241.

[14] _ Synthetic geometry in Riemannian manifolds, Proc. Internat. Congr. Math. (Helsinki, 1978), Acad. Sci. Fennica, Helsinki, 1980, 415-419.

[15] M. Gromov, J. Lafontaine \& P. Pansu, Structure métrique pour les variétés riemanniennes, Cedic/Fernand Nathan, Paris, 1981.

[16] K. Grove \& H. Karcher, How to conjecture $C^{1}$-close actions, Math. Z. 132 (1973) 11-20.

[17] A. Katsuda, Gromov's convergence theorem and its application, Nagoya Math. J. 100 (1985) 11-48.

[18] S. Kobayashi \& K. Nomizu, Foundations of differential geometry. I, Interscience, New York, 1963.

[19] H. B. Lawson \& S. T. Yau, Compact manifolds of nonpositive curvature, J. Differential Geometry 7 (1972) 211-228.

[20] S. Peters, Convergence of Riemannian manifolds, Compositio Math. 62 (1987) 3-16.

[21] E. Ruh, Almost flat manifolds, J. Differential Geometry 17 (1982) 1-14.

[22] W. Thurston, The geometry and topology of 3-manifolds, Princeton University, Princeton, $\mathrm{NJ}$.

[23] C. T. C. Wall, Surgery on compact manifolds, Academic Press, New York, 1970.

[24] T. Yamaguchi, Manifolds of almost nonnegative Ricci curvature, J. Differential Geometry 28 (1988) 157-167.

UNIVERSITY OF TOKYO

SAGa UNIVERSITY 